\title{
The European VLF/LF radio network to search for earthquake precursors: setting up and natural/man-made disturbances
}

\author{
P. F. Biagi ${ }^{1,2}$, T. Maggipinto ${ }^{1}$, F. Righetti ${ }^{1}$, D. Loiacono ${ }^{1}$, L. Schiavulli ${ }^{1}$, T. Ligonzo ${ }^{1}$, A. Ermini ${ }^{3}$, I. A. Moldovan ${ }^{4}$, \\ A. S. Moldovan ${ }^{5}$, A. Buyuksarac ${ }^{6}$, H. G. Silva ${ }^{7}$, M. Bezzeghoud ${ }^{7}$, and M. E. Contadakis ${ }^{8}$ \\ ${ }^{1}$ Department of Physics, University of Bari, Bari, Italy \\ ${ }^{2}$ Inter-Department Centre for the Evaluation and Mitigation of the Volcanic and Seismic Risk, University of Bari, Italy \\ ${ }^{3}$ Department of Mechanical Engineering, University of Tor Vergata, Rome, Italy \\ ${ }^{4}$ National Institute of Earth's Physics, Seismological Depth., Bucharest, Magurele, Romania \\ ${ }^{5}$ AZEL-Designing Group S.R.L., Bucharest, Magurele, Romania \\ ${ }^{6}$ Canakkale Onsekiz Mart University, Depth. of Geophysics, Canakkale, Turkey \\ ${ }^{7}$ Geophysical Centre of Évora and Physics Department, ECT, University of Évora, Portugal \\ ${ }^{8}$ University of Thessaloniki, Department of Surveying \& Geodesy, Thessaloniki, Greece
}

Received: 12 October 2010 - Revised: 16 December 2010 - Accepted: 25 December 2010 - Published: 7 February 2011

\begin{abstract}
In the last years disturbances in VLF/LF radio signals related to seismic activity have been presented. The radio data were collected by receivers located on the ground or on satellites. The ground-based research implies systematic data collection by a network of receivers. Since 2000 the "Pacific VLF network", conducted by Japanese researchers, has been in operation. During 2008 a radio receiver was developed by the Italian factory Elettronika (Palo del Colle, Bari). The receiver is equipment working in VLF and LF bands. It can monitor 10 frequencies distributed in these bands and, for each of them, it saves the power level. At the beginning of 2009, five receivers were made for the realization of the "European VLF/LF Network"; two were planned for Italy and one for Greece, Turkey and Romania, respectively. In 2010 the network was enlarged to include a new receiver installed in Portugal. In this work, first the receiver and its setting up in the different places are described. Then, several disturbances in the radio signals related to the transmitters, receivers, meteorological/geomagnetic conditions are presented and described.
\end{abstract}

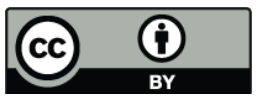

Correspondence to: P. F. Biagi

(biagi@fisica.uniba.it)

\section{Introduction}

Processes that occur during the preparation phase of a forthcoming earthquake, such as gas and ionized particles emission from the Earth's surface, fluctuations in the groundwater level, changes in the electrical resistivity of the ground, give rise to phenomena such as micro-variations in the Earth's gravity, electromagnetic emissions and changes in the refractive index of the troposphere. According to the most recent and convincing models (Mareev et al., 2002; Molchanov, 2004), these phenomena determine a particular lithosphereionosphere coupling and cause variation of the medium in which radio signals propagate, affecting especially radio propagation in the VLF $(15-60 \mathrm{kHz})$ and LF $(150-300 \mathrm{kHz})$ bands. The VLF radio signals are used for time signal, navigation and military purposes and they propagate using the Earth-ionosphere channel as a waveguide. The LF radio signals are used in long-wave broadcasting and are characterized by a ground-wave and a sky-wave propagation mode. The first generates a stable signal that propagates in the channel Earth-troposphere; the second generates a signal which varies greatly between day and night, summer and winter, and which propagates using the lower ionosphere as a reflector. In any case, the electric field strength (intensity) of the VLF and LF radio signals is high at nighttime and low at daytime. 
Table 1. List of the VLF/LF radio signals sampled by each receiver of the network.

\begin{tabular}{lll}
\hline Receiver & \multicolumn{2}{c}{ VLF } \\
& \multicolumn{1}{c}{ LF } \\
\hline IT-Ba & GBZ, ICV, DHO, NRK, ITS & \\
IT-An & GBZ, HWU, DHO, NRK & FRI, TRT, EU1, MCO, RRU, CZE \\
IT-Tc & GBZ, ICV, DHO, NRK, ITS & RRO, TRT, EU1, MCO, CZE \\
GR & GBZ, ICV, DHO, NRK, ITS & RRO, TRT, EU1, CH1, CZE \\
TUR & GBZ, ICV, HWU, DHO, ITS & RRO, TRT, EU1, RRU, CZE \\
ROM & GBZ, GBZ, HWU, DHO, NRK, ITS & EU1, CH1, MCO, CZE \\
POR & GBZ, HWU, DHO, NRK, ITS & RRO, TRT, EU1, CH1, MCO \\
\hline
\end{tabular}

During the last few years, disturbances in VLF/LF radio signals related to seismic activity have been presented (Biagi et al., 2001a, b; Biagi and Hayakawa, 2002; Biagi et al., 2004, 2005, 2006, 2007; Gufeld et al., 1992; Hayakawa and Sato, 1994; Hayakawa et al., 1996, 2006; Molchanov and Hayakawa, 1998; Rozhnoi et al., 2005, 2006a, b). The radio data were collected by receivers located on the ground. Recently, some possible seismic disturbances revealed by VLF radio signals collected on board the French DEMETER satellite were presented by Molchanov et al. (2006) and Rozhnoi et al. (2007).

Ground-based research is carried out by means of receivers able to sample some parameters of the radio signals as intensity and phase with a rate of few seconds or minutes. Here, the setting up of a new network installed in Europe at the beginning of 2009 is described. Several disturbances pointed out during the first running period are presented and discussed.

\section{Radio receivers and networks}

Since 2000 Japanese researchers have been relying on the existence of a VLF radio network (Pacific network) of seven receivers able to measure the intensity and the phase of VLF radio signals from two different transmitters (Fig. 1a). The receivers are the Japanese OmniPal model (Dowden and Adams, 1989) and are connected to a computer through a digital card and use a GPS sensor and a rectilinear antenna for the signal's registration (Fig. 1b).

On February 2002, within the framework of a scientific cooperation among Japanese, Russian and Italian teams, an OmniPal receiver, labelled IT-Ba, was put into operation in the Department of Physics of the University of Bari (south Italy, Fig. 2), giving input to the development of a European network. On the basis of the best reception at the receiver and, taking into account the most convenient radio paths in relation to the seismic activity, the following transmitters were selected: GBZ, ICV, DHO, NRK and ITS (Table 1). Their locations are shown in Fig. 2 and some of their peculiarities are reported in Table 2. The sampling rate used is
Table 2. Peculiarities of the VLF/LF transmitters of the European network.

\begin{tabular}{llcc}
\hline Label & Country & Power $(\mathrm{kW})$ & Frequency $(\mathrm{kHz})$ \\
\hline GBZ & Great Britain & & $19.58-22.10$ \\
ICV & Sardinia (Italy) & & 20.27 \\
HWU & France & & 21.75 \\
DHO & Germany & & 23.4 \\
NRK & Iceland & & 37.5 \\
ITS & Sicily (Italy) & & 45.9 \\
RRO & Romania & 1200 & 153 \\
FRI & France & 2000 & 162 \\
TRT & Turkey & 1200 & 180 \\
EU1 & Germany & 2000 & 183 \\
CH1 & Algeria & 2000 & 198 \\
MCO & France & 1200 & 216 \\
RRU & Russia & 2500 & 261 \\
CZE & Czech Republic & 500 & 270 \\
\hline
\end{tabular}

$1 \mathrm{~min}$. At the same site a meteorological station was put into operation during 2005.

During 2008 a new receiver was developed by the Italian factory Elettronika (Palo del Colle, Bari). The new receiver can work both in the VLF and the LF band. It can monitor a total of 10 frequencies distributed in these bands and, for each of them, it saves the power level detected on a big nonvolatile memory at a selectable sample time interval. The power level is expressed in $\mathrm{dB}_{m}$ as $\mathrm{dB}_{m}=20 \log \left(\mathrm{V}_{\mathrm{mVpp}}\right)$. For simplicity, in the different figures of this paper showing data collected with this receiver, the $\mathrm{dB}_{m}$ indication on the $\mathrm{y}$-axis is not reported.

The receiver has two standard XLR antenna connectors, one for each band, with four poles. On the same connector there is the differential input signal from the antenna and the dual power supply voltage for the preamplifier that is located near the antenna. Its aim is to convert the high impedance of the low-frequency antennas to a low impedance to drive the cable and to amplify the captured signal level introducing very low noise. The two antennae, made of brass, are 

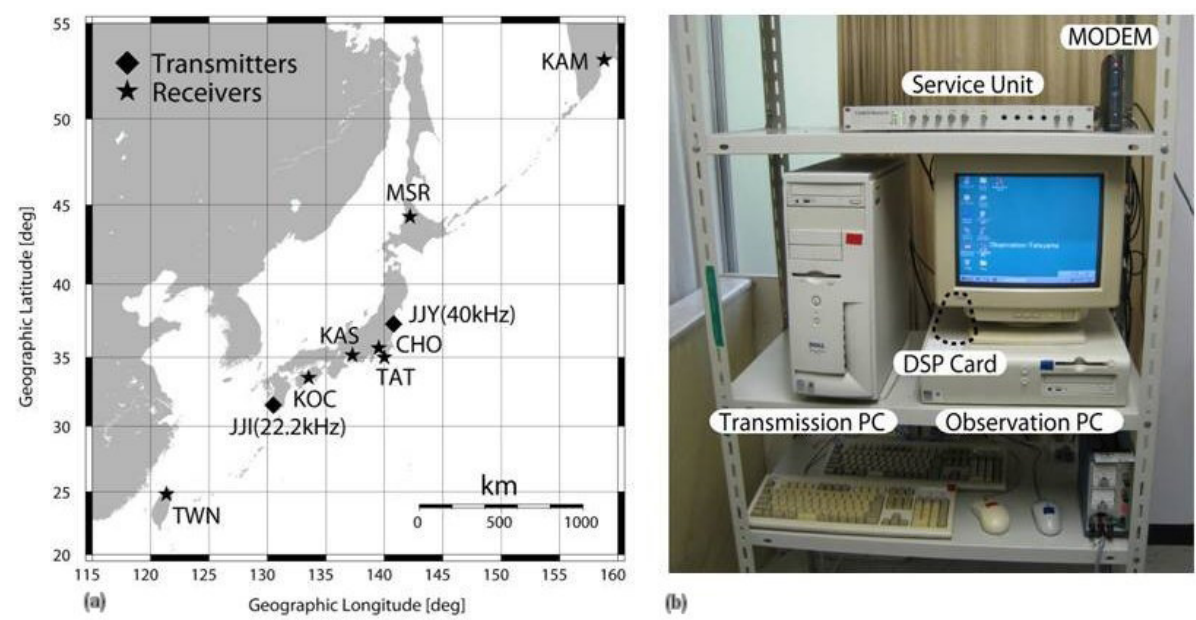

Fig. 1. (a) Pacific VLF radio network. The receivers (OmniPal) are marked by stars and five are located in Japan, one in Kamchatka (Russia), and one in Taiwan. The signals collected come from JJY ( $40 \mathrm{kHz})$ and JJI $(22.2 \mathrm{kHz})$ transmitters located in Japan. (b) The OmniPal recording system.

rectilinear, long $1.5 \mathrm{~m}$ (LF band) and $2.0 \mathrm{~m}$ (VLF band), respectively. The huge amount of data collected is organized in text files, one for each day. The configuration of the equipment, the check of its status and the download of the collected data are made possible using resident software with a Web-based Graphical User Interface and of an HTTP server. Data transfer and management is also possible through a resident FTP server. Then, either local or remote TCP/IP connectivity with the instrument and above-mentioned software is allowed by means of a standard Ethernet interface and of a GSM modem (configured for data calls), both mounted in the chassis of the receiver. The equipment is represented on the home page of the INFREP (International Network for Frontier Research on Earthquake Precursors) web site (http://beta.fisica.uniba.it/infrep/).

It should be mentioned that the receiver was designed for easy interactivity and communication. Thanks to the facility of remote and furthermore "web-ready" data management, the set up of a network of such receivers, located on strategic sites (from a seismic point of view), was straightforward. In fact, at the beginning of 2009 five receivers were prepared, two for Italy and one for Greece, Turkey and Romania, respectively, and the setting-up started. In 2010 another Elettronika receiver was installed in Portugal.

At the moment all data from the various sites are collected on a weekly basis in the Department of Physics in Bari and are processed for suitable analysis.

However, in order to find the best reception, several actions were made in the different places, as is described in the next section. The receivers are labelled IT-An and ITTc (Italy), GRE (Greece), TUR (Turkey), ROM (Romania), POR (Portugal) and their actual locations are shown in Fig. 2. A sampling rate of $1 \mathrm{~min}$ was set for all receivers.

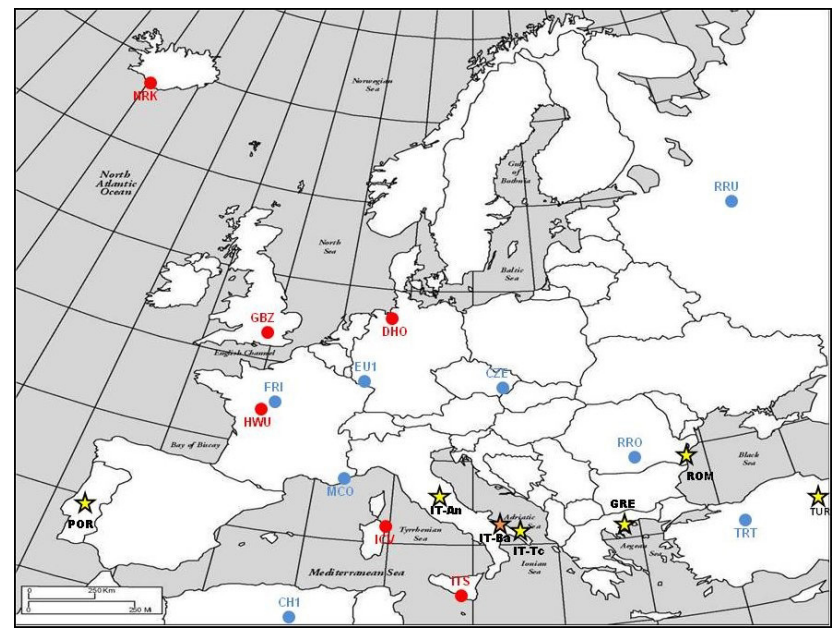

Fig. 2. Map showing the receivers and transmitters of the network. The stars show the locations of the VLF/LF receivers. The orange star represents the OmniPal type; the yellow ones represent the Elettronika type. The circles on the map indicate the LF (blue) and VLF (red) transmitters, the signals of which are collected by the different receivers. Some peculiarities of these transmitters are reported in Table 2.

Some peculiarities of the VLF and LF transmitters, the signal of which is sampled by the various receivers (as specified in next section), are reported in Table 2. Their locations are shown in Fig. 2.

\section{Setting up the Elettronika receivers}

The setting up of the first five receivers was carried out from March to July 2009. The setting up of the receiver in Portugal is still in progress. The main effort was: (a) the sampling 


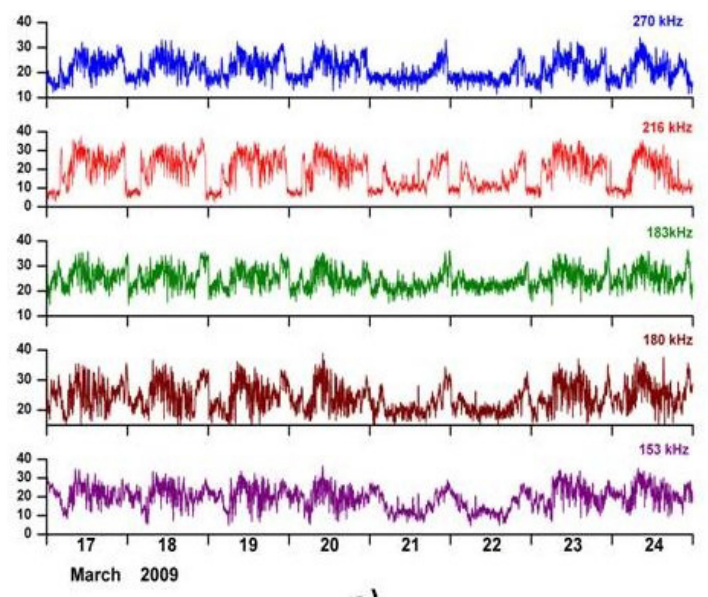

a)
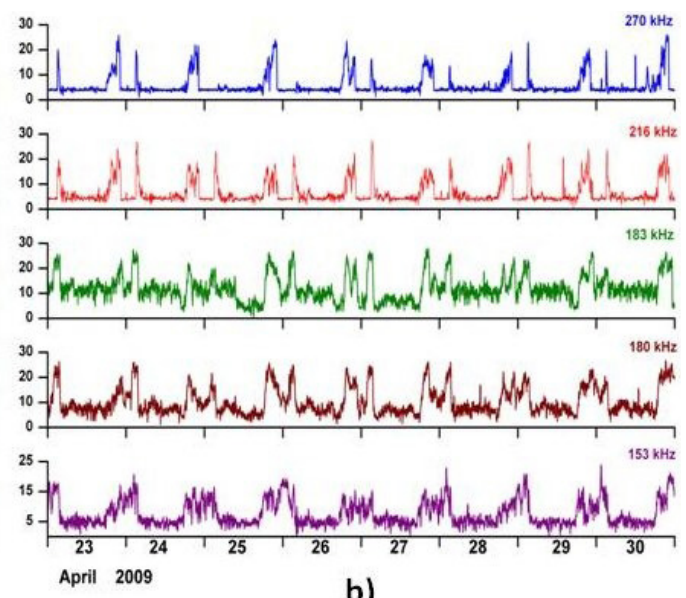

b)

Fig. 3. (a) Trend of the intensity of five LF radio signals sampled in Bari (southern Italy) during a period of eight days. The noise appears reduced on 21-22 March, corresponding to Saturday and Sunday. (b) Trend of the intensity of the same radio signals sampled in Torre Canne (southern Italy) during a period of eight days. A low level of noise is evident in each trend during all days.

of the same radio signals by different receivers; (b) the reception with a low noise level; (c) the reliability of the on-line connection. Sometimes in order to realize the items (b) and (c), it has not been possible to respect the statement (a). The situation at each site is described in the following.

- IT-An: At the beginning the receiver was installed near the mouth of the Amare cave that is situated on the southern slope of the Gran Sasso mountain chain, at a distance of $10 \mathrm{~km}$ from L'Aquila city (central Italy). Since 1985, in order to investigate possible earthquake precursors, some of the authors have installed a receiver in the same place, powered by a battery connected to a solar panel, able to measure the intensity of three LF radio signals and the Electronika equipment was planned to substitute for this one. The main problem of the new receiver in this site is its power consumption, which is quite high $(20 \mathrm{~W})$, and the difficulty of the online connection (low mobile phone field). After different attempts, the decision was made to move the receiver to another site; a country house in a small village located at a distance of about $20 \mathrm{~km}$ west of the previous one was available and here the receiver was put into operation. The location of the site is shown in Fig. 2 and the list of the radio signals actually sampled is reported in Table 1.

- IT-Tc: At the beginning the receiver was installed in Bari (Department of Physics of the University) with the purpose of substituting for the OmniPal receiver in operation here. Unfortunately, the reception of the LF signals (not sampled by the OmniPal receiver) appeared extremely disturbed because of a high noise level. The situation is shown in Fig. 3a where it is evident that acceptable reception exists only at the weekends. Due to the fact that the reception of the VLF signals is good in this place, the decision was to leave the Omnipal receiver for VLF signals sampling here and to move the Elettronika receiver to another place. A house in a village (Torre Canne), located south of Bari city at about $80 \mathrm{~km}$, was available and the receiver was put into operation here. The location of this site is shown in Fig. 2. Here, a good reception of the LF radio signals exists as Figure $3 \mathrm{~b}$ shows. The radio signals actually sampled in this place are indicated in Table 1.

- GR: The receiver was put into operation in the building of the School of Art in the University of Thessaloniki. Some frequencies were changed in order to have better reception and radio paths crossing more seismic zones. The radio signals actually sampled in this place are indicated in Table 1 and the location of the site is shown in Fig. 2.

- TUR: At the beginning the receiver was put into operation in Sivas city (Cumhuriyet University). A high noise level both for VLF signals and for LF signals appeared, as shown in Fig. 4a. Several tests have revealed that the noise was the electrical background of the city, so the decision was made to move the receiver to another "quieter" site. Some tests have indicated the thermal Resadiye zone, in the North Anatolian Fault Zone, located at about $70 \mathrm{~km}$ north of Sivas, as a suitable site for good reception of the radio signals. A hotel in this zone was available and here the receiver was put into operation. The excellence of the reception appears in Fig. 4b. The location of the site is shown in Fig. 2. The list of the signals actually sampled is reported in Table 1 . 

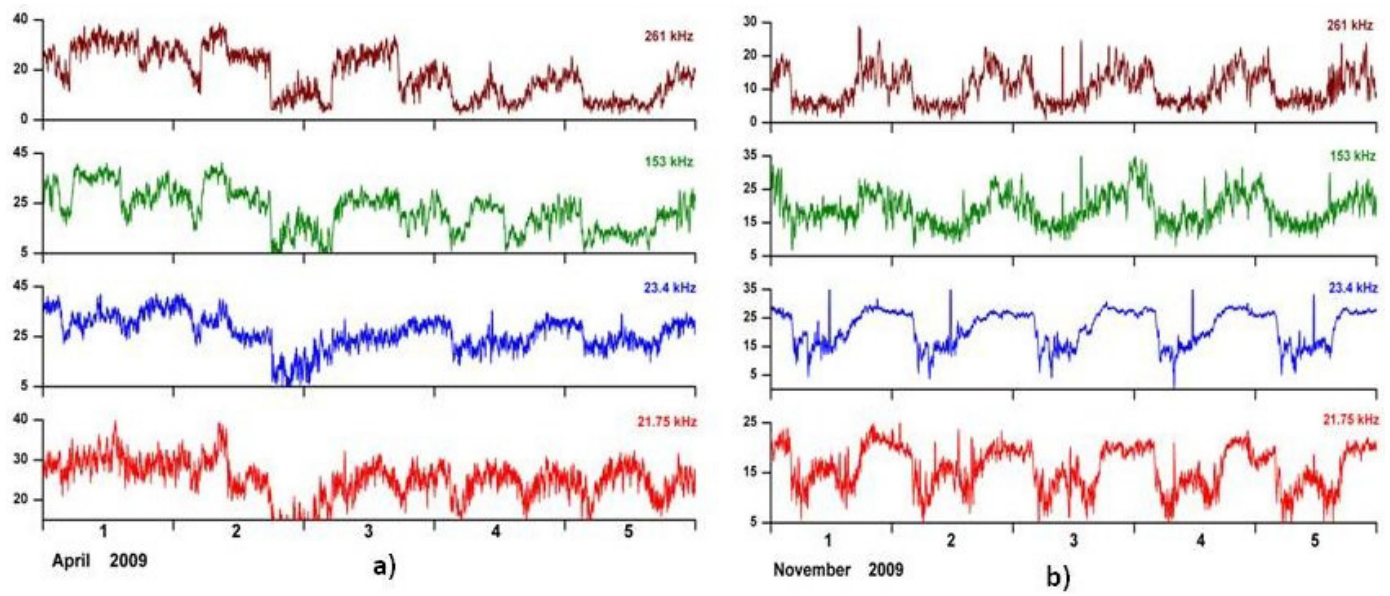

Fig. 4. (a) Trend of the intensity of four VLF/LF radio signals sampled in Sivas (eastern Turkey) during five days. All the signals appear disturbed and irregular. (b) Trend of the intensity of the same radio signals sampled in Resadiye (eastern Turkey) during five days. The reception of the different signals is good enough and regular.

- ROM: After some tests in Bucharest, the receiver was moved to the Black-Sea shore (Dobruja Seismologic Observatory). The location is indicated in Fig. 2. Here, at beginning, unsatisfactory data characterized by a high noise-level on both the bands were obtained. The whole receiving chain was verified using a programmable signal generator and other specific lab-testing devices. The results of this check-up revealed that there was no malfunction of the receiver due to damage or something similar. Then, it was noted that the monitored data did not show any diurnal variation of the electric field's intensities at the chosen frequencies. Moreover, the output data indicated a high level of the electric field's intensities at all frequencies, as there was a large spectrum parasitic electric field. This behavior could be generated either by the high-voltage aerial lines surrounding the observatory or by the local specific electric proprieties of the atmosphere, which is salt-saturated. After some tests, the decision was not to the change of the site (because it represents a good place for this research) but the replacement of the rectilinear electric-type antennas with loop magnetic-type ones. These new antennas were planned and realized by the Romanian Team and they are described in detail in Moldovan et al. (2010). The change has enabled good reception of both the VLF signals and the LF ones, as shown in Fig. 5. The list of the radio signals actually sampled in this site appears in Table 1.

- POR: The Elettronika receiver was put into operation in the University of Évora. Évora is a city located about $100 \mathrm{~km}$ in west direction respect to Lisbon. The location is shown in Fig. 2. The setting up is still in progress; the radio signals actually sampled are indicated in Table 1 .

\section{Main disturbances of radio data}

The research of seismic effects on the radio data is based on the spotting of disturbances. In this framework a fundamental step is the identification of possible disturbances related to causes different than seismicity. The analysis of data collected by the receivers of the network has allowed us to reveal several disturbances of this type. They appear to be related to the transmitter, to the receiver or to meteorologi$\mathrm{cal} /$ geomagnetic conditions.

\subsection{Disturbances related to the transmitter}

Figure 6 shows a drop of about five days in the intensity of the DHO radio signal. The drop appears simultaneously in the data collected by all the receivers in operation at that time (August, 2009). It is evident that this disturbance is related to some interruption of the normal broadcast of the DHO radio station.

Figure 7 shows a change in the trend of the HWU intensity collected by the IT-An, TUR and ROM receivers starting from 5 July 2010. The quality of reception increases strongly at ROM site where a bad signal becomes a good one. A little improvement happens at IT-An site, whereas the reception becomes bad at TUR site where an electronic saturation takes place; in fact the intensity of the signal is lower at nighttime than at daytime, i.e. the situation is opposite to the normal one. The disturbances shown in Fig. 7 are clearly connected with an increase of the power radiated from the HWU transmitter. Similarly, disturbances connected with decrease of the radiated power from some transmitters have been observed and, for example, a good signal recorded in a place can thus become a bad signal. 

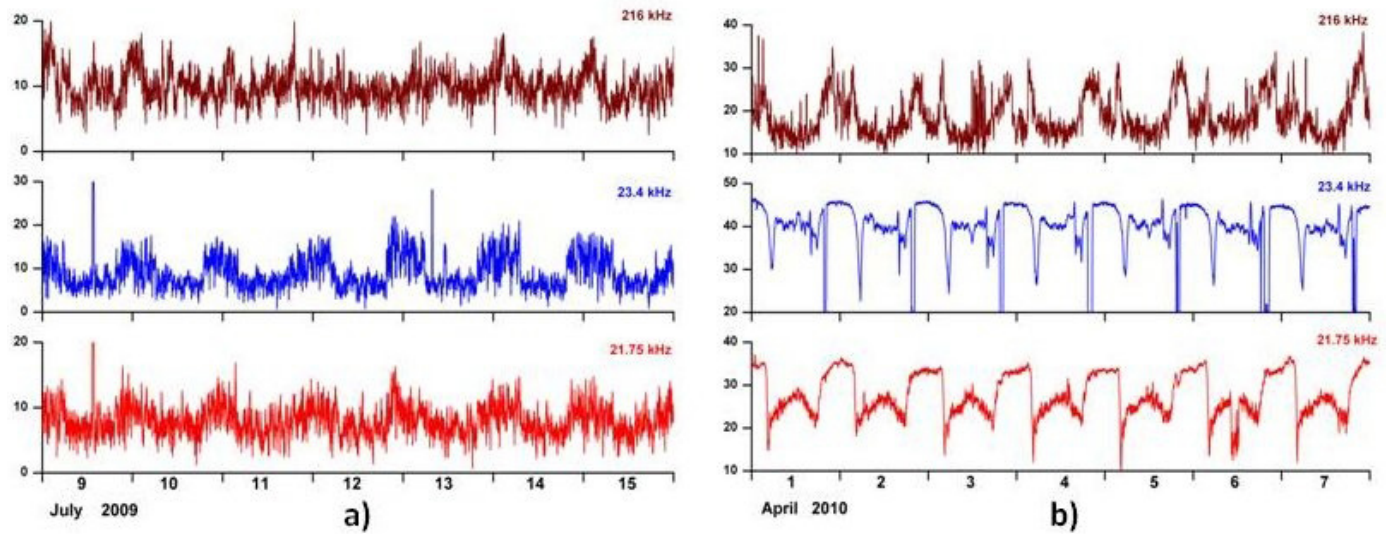

April 2010 b)

Fig. 5. (a) Trend of the intensity of VLF/LF radio signals sampled during seven days in Dobruja Observatory (Romania) using the rectilinear electric antennae. All the signals appear disturbed and irregular. (b) Trend of the intensity of the same radio signals sampled in the same place during seven days using the loop magnetic antennae. The reception of the different signals appears good enough and regular.
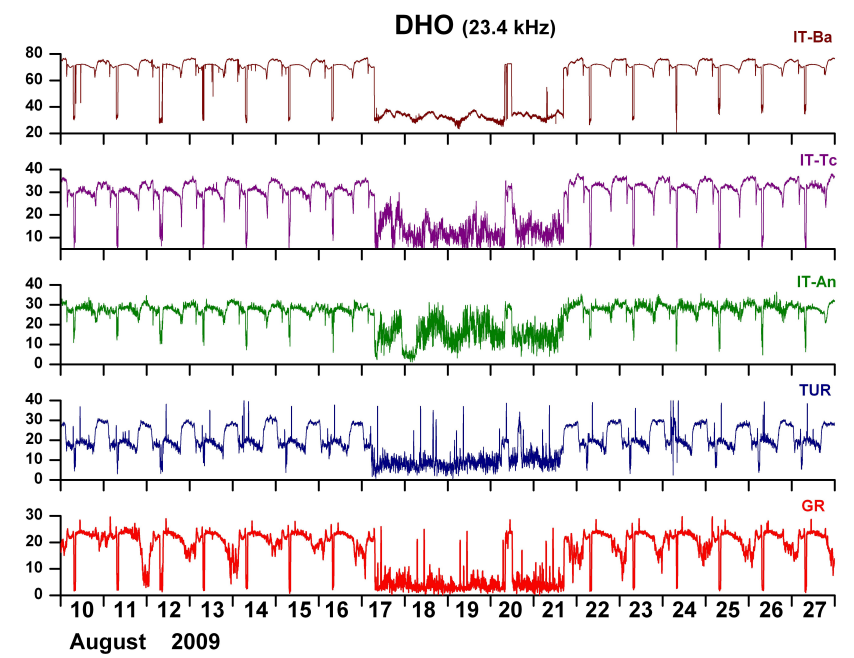

Fig. 6. Trends of the intensity of DHO $(23.4 \mathrm{kHz})$ radio signal sampled by IT-Ba, IT-Tc, IT-An, TUR and GR receivers during 10-27 August 2009. A drop in the values in the time interval 17-21 August clearly stands out.

\subsection{Disturbances related to the receiver}

Figure 8 shows a decrease of the intensity of VLF radio signals collected by IT-Tc receiver, starting on 8 January 2010 . No similar effect appears on the LF radio signals collected by the same receiver; at the same time, the VLF radio signals collected in the other sites of the network do not show the same decrease. A control of the IT-Tc equipment carried out after several days, has revealed a breaking of the VLF antenna, probably due to a gust of wind. After the antenna was repaired, the effect disappeared.

Figure 9 shows an increase/disturbance of the intensity of both LF and VLF radio signals collected by the IT-Tc re-

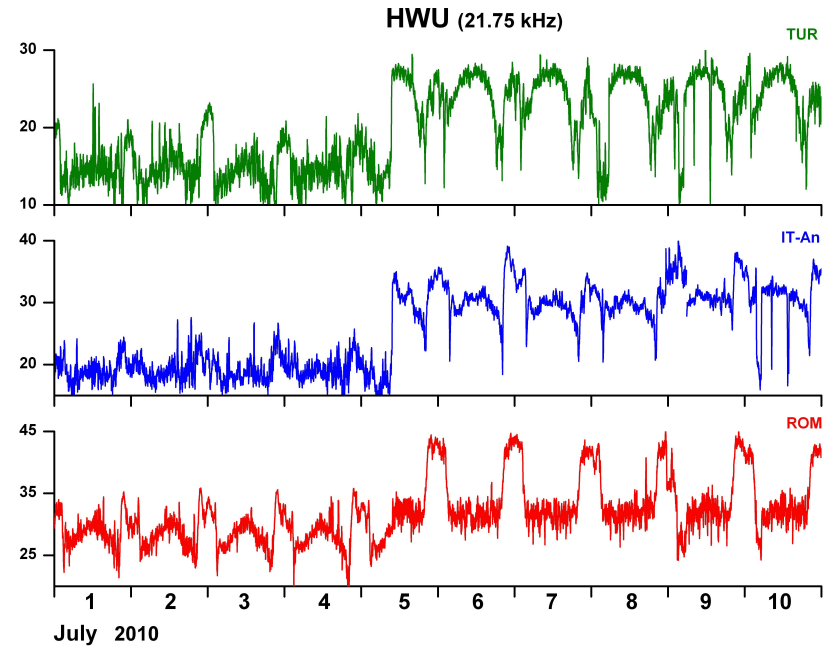

Fig. 7. Trends of the intensity of the HWU $(21.75 \mathrm{kHz})$ radio signal collected by IT-An, TUR and ROM receivers during 1-10 June, 2010. Starting from 5 July, a change in all the trends appears. At ROM the quality of reception increases clearly; a little improvement happens at IT-An, while the reception turns bad at TUR, where the signal becomes saturated.

ceiver in the time interval 27-30 July, 2009. Different controls carried out at the site enabled us to discover that the increase/disturbance was produced by a radio amateur operating in a house located near the receiver.

\subsection{Disturbances related to meteorological/geomagnetic conditions}

Figure 10 shows the trends of the intensity of two LF and two VLF radio signals collected by the IT-Tc receiver in the time interval 9-18 October 2009. In the same figure the air temperature, the air pressure and the rainfall recorded by the 


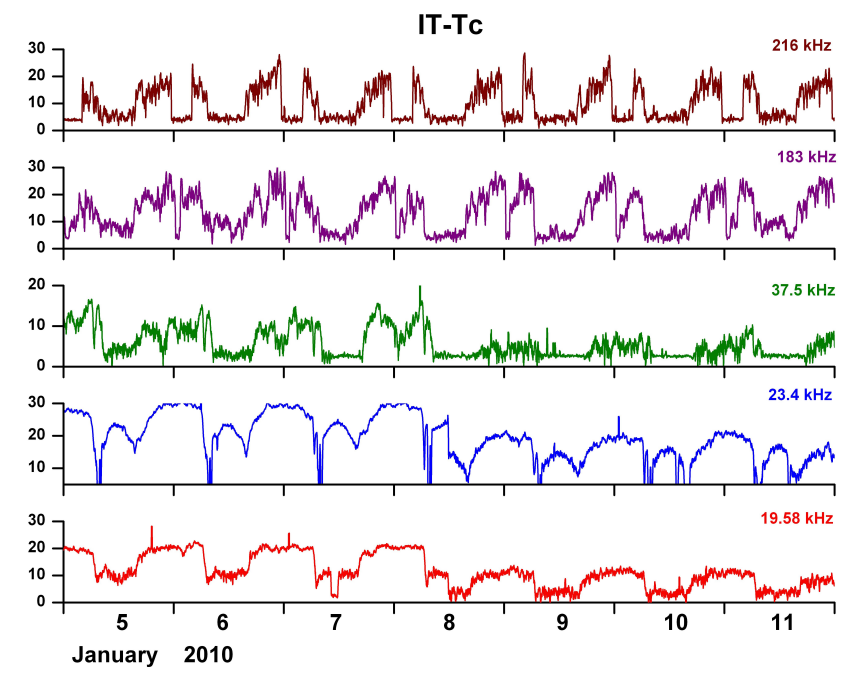

Fig. 8. Trends of the intensity of the MCO $(216 \mathrm{kHz})$, EU1 $(183 \mathrm{kHz}), \mathrm{NRK}(37.5 \mathrm{kHz}), \mathrm{DHO}(23.4 \mathrm{kHz})$ and GBZ $(19.58 \mathrm{kHz})$ radio signals collected by IT-Tc receiver during 5-11 January, 2010. Starting from January 8, a decrease appears on VLF (NRK, DHO, GBZ) signals, while no modification appears on LF (MCO, EU1) signals.

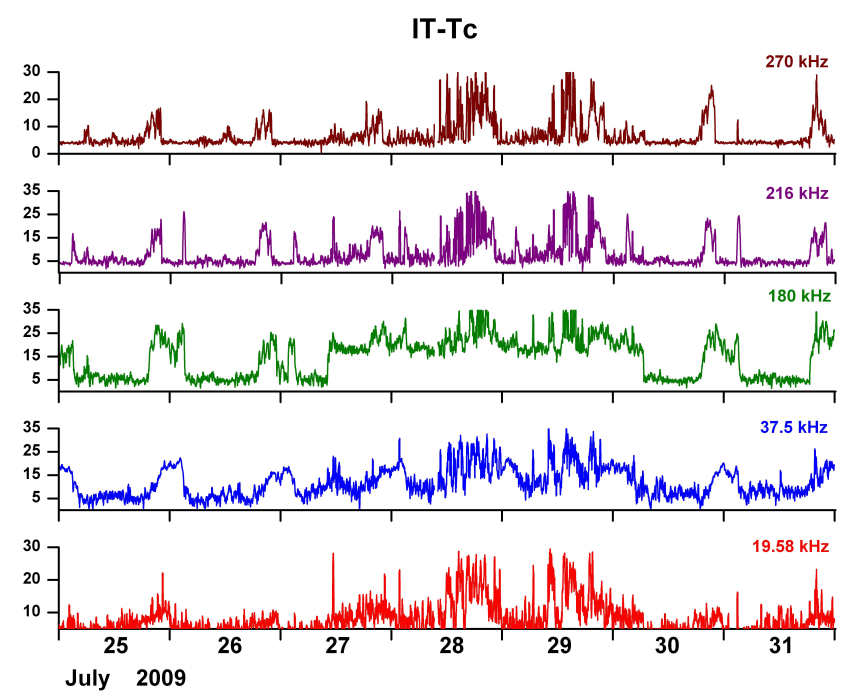

Fig. 9. Trends of the intensity of the CZE $(270 \mathrm{kHz}), \mathrm{MCO}$ $(216 \mathrm{kHz})$, TRT $(180 \mathrm{kHz}), \mathrm{NRK}(37.5 \mathrm{kHz})$ and GBZ $(19.58 \mathrm{kHz})$ radio signals collected by IT-Tc receiver during 25-31 July, 2009. From 27 to $30 \mathrm{July,}$ an increase/disturbance happens on all the trends.

meteorological station operating in Bari (Sect. 2) is reported. From the figure a decrease/disturbance of the radio intensities appears on 13-14 October; during those same days the meteorological data reveal an abrupt decrease of air temperature, a large decrease of air pressure and the occurrence of rain. This meteorological situation is related to Bari, but local information indicated that the meteorological situation in

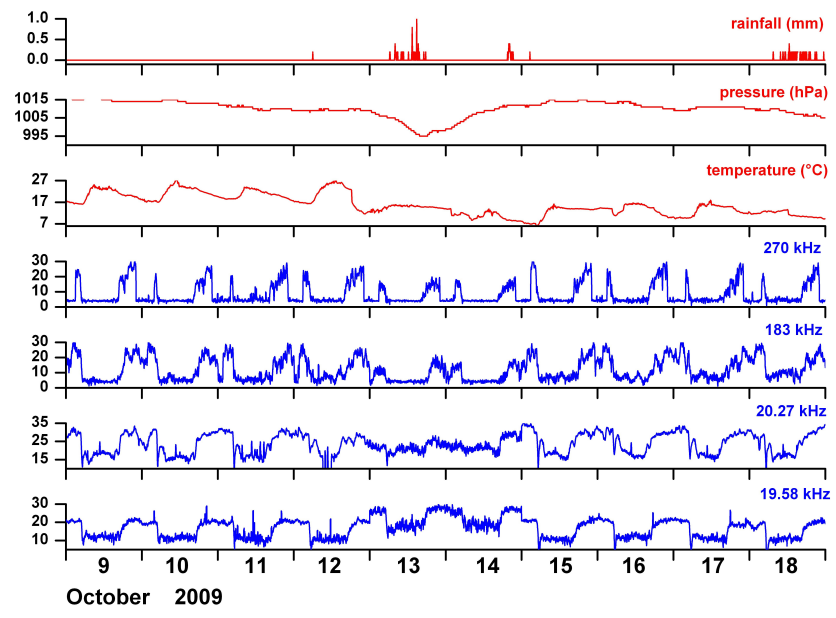

Fig. 10. The three upper panels represent the air temperature, air pressure and rainfall in Bari in the time interval 9-18 October 2009. The remaining panels show the trends of the intensity of CZE $(270 \mathrm{kHz})$ and TRT $(183 \mathrm{kHz}) \mathrm{LF}$ radio signals and of ICV $(20.27 \mathrm{kHz})$ and GBZ $(19.58 \mathrm{kHz})$ VLF radio signals collected by IT-Tc receiver during the same period. A decrease/disturbance of the radio intensities appears during 13-14 October when an abrupt decrease of the air temperature, a large decrease of the air pressure and rain happened.

Torre Canne, that is the place where the receiver IT-Tc is into operation, was more intense and crucial. So, the possibility that the decrease/disturbance of the radio intensities at IT-Tc is related to the meteorological condition seems realistic. In any case, it must be noted that the radio data at IT-Ba do not reveal any clear effect.

Figure 11 shows the trends of the daytime intensity (the range from 09:00 a.m. to 02:00 p.m. (UT) was selected) of RRO, TRT, EU1, MCO radio signals collected by the GR receiver in the time interval 17-23 June 2010. An evident increase of intensity appears on all the trends during June 20. On this day, from the local meteorological data the occurrence of an intense summer storm stands out. Hence, the radio intensity increases pointed out are connected to the electricity produced by lightning.

Figure 12 shows the intensity of GBZ, DHO, NRK radio signals collected by the IT-Ba receiver (Omnipal type) in the time interval 1-20 November 2004. In the same figure the geomagnetic indices $D_{s t}$ and $k_{p}$ are reported. In the days from 7 to 11 November, disturbances appear on the radio intensities; very clear is the effect on the DHO signal. Simultaneously, the geomagnetic indices reveal a large increase of geomagnetic activity. So, it is very realistic that the disturbances on the radio signals were produced by the geomagnetic condition. 

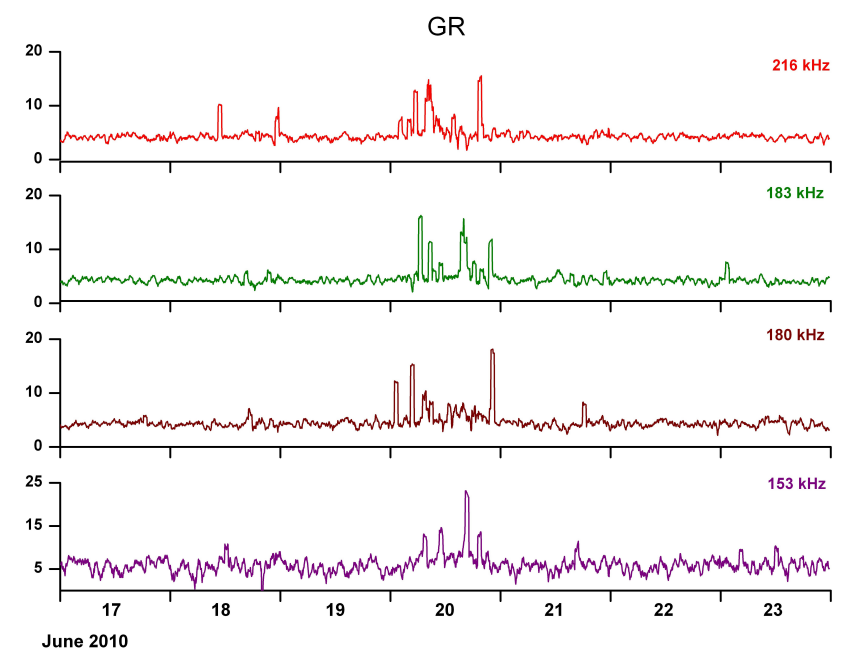

Fig. 11. Daytime intensity of the RRO $(153 \mathrm{kHz})$, TRT $(180 \mathrm{kHz})$, EU1 $(183 \mathrm{kHz})$ and MCO $(216 \mathrm{kHz})$ radio signals collected by the GR receiver during 17-23 June 2010. The data are copied by the raw set selecting each day the time interval from 09:00 a.m. to 02:00 p.m. (UT). An evident increase appears on all the trends on 20 June, a day characterized by an intense summer storm.

\section{Discussion}

Meteorological conditions and geomagnetic activity can affect the propagation of VLF/LF radio signals. Particularly, the meteorological conditions produce disturbances in the troposphere refractive index, troposphere electricity (lightning), the relative permittivity and the conductivity of the ground; the geomagnetic activity produces variations in the ionosphere conditions. In the past, some disturbances on the propagation of the VLF/LF radio signals related to these effects have been presented (Kikuchi, 1981; Kleimenova et al., 2004; Biagi et al., 2005; Rozhnoi et al., 2006b). Mainly, meteorology affects the LF radio signals and the geomagnetic activity the VLF ones. The results presented in Figs. 10-12 confirm this influence. Anyway, if a correlation is claimed between radio disturbances and the meteorologi$\mathrm{cal} /$ geomagnetic variations, they must occur simultaneously. So, simultaneity is one of the criteria to individuate these radio disturbances.

Nonetheless, this study has revealed disturbances in radio signals related to variations of the power radiated by the transmitters, to some breaking of the receiver and to some human activity at a receiver site. The LF radio signals seem more disturbed than the VLF ones.

If the effect is related to the transmitter, the disturbance must appear simultaneously in the related signal recorded by the different receivers of the network. The cases shown in Figs. 6 and 7 are evident examples of this effect. So, this type of radio disturbance can be easy identified. It must be noted that a network of several receivers is fundamental for this purpose.

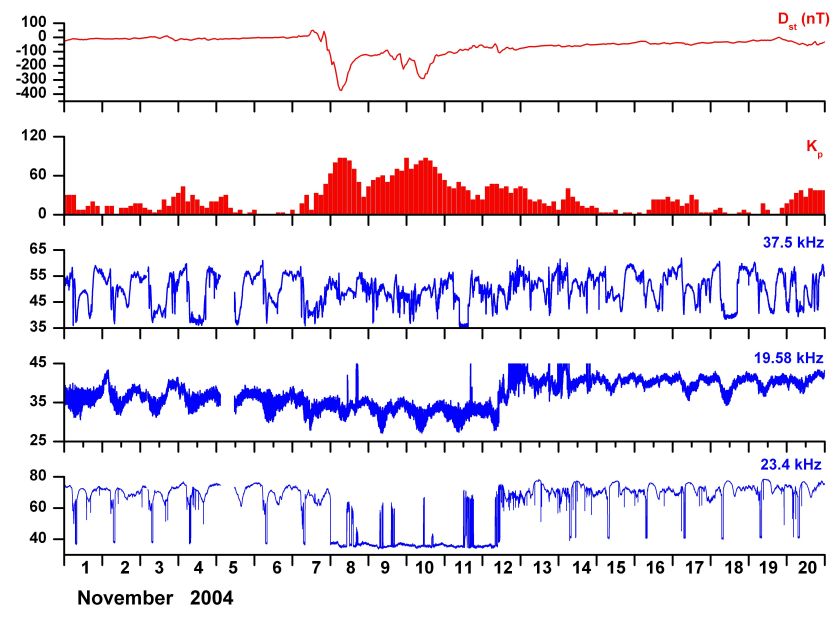

Fig. 12. In the period 1-20 November 2004, from the top reading downwards: $\mathrm{D}_{\mathrm{st}}$ geomagnetic index, $\mathrm{k}_{p}$ geomagnetic index, the intensity $(\mathrm{dB})$ of GBZ $(19.58 \mathrm{kHz})$, DHO $(23.4 \mathrm{kHz})$, NRK $(37.5 \mathrm{kHz})$ radio signals sampled by the IT-Ba receiver (Omnipal type). A large increase in the geomagnetic activity stands out during 7-11 November.

In the other two circumstances mentioned above, probably only the simultaneous appearance of the disturbance in all or several VLF and/or LF signals recorded by that receiver will allow the identification of its origin. The Figs. 8 and 9 show examples of these effects.

\section{Conclusions}

The study we performed has revealed that a lot of different sources of disturbances must be monitored in order to claim the disturbances in radio signals are related to seismicity. The next step of our research will be the study of the different time series of the radio data using methods of analysis such as the Wavelet, the PCA (Principal Component) and so on, in order to reveal anomalies in the radio signals related to the occurrence of earthquakes. The results of the present study will have a fundamental role in discriminating possible seismic effects from other different sources of disturbances.

Acknowledgements. This research was partially supported by Foundation of the Cassa di Risparmio di Puglia bank (F.C.R.P., Bari, Italy). The Portuguese team acknowledges gratefully the support of QREN (Operational Program "Thematic Factors of Competitiveness"), co-funded by the European Regional Development Fund (ERDF) for the research program SIRAS, and the FCT (Science and Technology Foundation) for the grant SFRH/BPD/63880/2009 (HGS).

Edited by: K. Eftaxias

Reviewed by: O. A. Pokhotelov and another anonymous referee 


\section{References}

Biagi, P. F., Piccolo, R., Ermini, A., Martellucci, S., Bellecci, C., Hayakawa, M., Capozzi, V., and Kingsley, S. P.: Possible earthquake precursors revealed by LF radio signals, Nat. Hazards Earth Syst. Sci., 1, 99-104, doi:10.5194/nhess-1-99-2001, 2001a.

Biagi, P. F., Piccolo, R., Ermini, A., Martellucci, S., Bellocci, C., Hayakawa, M., and Kingsley, S. P.: Disturbances in LF radio-signals as seismic precursors, Annali di Geofisica, 44, 5/6, $2001 b$.

Biagi, P. F. and Hayakawa, M., Possible premonitory behaviour of LF radiowaves on the occasion of the Slovenia earthquakes (M=5.2-6.0-5.1) occurred on March-May 1998, in: Seismo Electromagnetics: Lithosphere-Atmosphere-Ionosphere Coupling, (edited by: Hayakawa, M. and Molchanov, O.), TERRAPUB, Tokyo, 249-253, 2002.

Biagi, P. F., Piccolo, R., Castellana, L., Maggipinto, T., Ermini, A., Martellucci, S., Bellecci, C., Perna, G., Capozzi, V., Molchanov, O. A., Hayakawa, M., and Ohta, K.: VLF-LF radio signals collected at Bari (South Italy): a preliminary analysis on signal anomalies associated with earthquakes, Nat. Hazards Earth Syst. Sci., 4, 685-689, doi:10.5194/nhess-4-685-2004, 2004.

Biagi, P. F., Castellana, L., Maggipinto, T., Piccolo, R., Minafra, A., Ermini, A., Martellucci, S., Bellecci, C., Perna, G., Capozzi, V., Molchanov, O. A., and Hayakawa, M.: A possible preseismic anomaly in the ground wave of a radio broadcasting $(216 \mathrm{kHz})$ during July-August 1998 (Italy), Nat. Hazards Earth Syst. Sci., 5, 727-732, doi:10.5194/nhess-5-727-2005, 2005.

Biagi, P. F., Castellana, L., Maggipinto, T., Piccolo, R., Minafra, A., Ermini, A., Martellucci, S., Bellecci, C., Perna, G., Capozzi, V., Molchanov, O. A., and Hayakawa, M.: LF radio anomalies revealed in Italy by the wavelet analysis: Possibile preseismic effects during 1997-1998, Phys. Chem. Earth, 31, 403-408, 2006.

Biagi, P. F., Castellana, L., Maggipinto, T., Maggipinto, G., Minafra, A., Ermini, A., Capozzi, V., Perna, G., Solovieva, M., Rozhnoi, A., Molchanov, O. A., and Hayakawa, M.: Decrease in the electric intensity of VLF/LF radio signals and possible connections, Nat. Hazards Earth Syst. Sci., 7, 423-430, doi:10.5194/nhess-7-423-2007, 2007.

Dowden, R. L. and Adams, C. D., Phase and amplitude perturbations on the NWC signal at Dunedin form lightning-induced electron percipitation, J. Geophys. Res., 94, 497-503, 1989.

Gufeld, I. L., Rozhnoi, A. A., Tyumensev, S. N., Sherstuk, S. V., and Yampolsky, V. S.: Radiowave disturbances in period to Rudber and Rachinsk earthquakes, Phys. Solid Earth, 28, 3, 267-270, 1992.

Hayakawa, M. and Sato, H.: Ionospheric perturbations associated with earthquakes, as detected by subionospheric VLF propagation, in: Electromagnetic Phenomena Related to Earthquake Prediction, (edited by: Hayakawa, M. and Fujinawa, Y.), TERRAPUB, Tokyo, 391-397, 1994.
Hayakawa, M., Molchanov, O. A., Ondoh, T., and Kawai, E.: The precursory signature effect of the Kobe earthquake on subionospheric VLF signals, J. Comm. Res. Lab., 43, 169-180, 1996.

Hayakawa, M., Ohta, K., Maekawa, S., Yamauchi, T., Ida, Y., Gotoh, T., Yonaiguchi, N., Sasaki, H., and Nakamura, T.: Electromagnetic precursors to the 2004 Mid Niigata Prefecture earthquake, Phys. Chem. Earth, 31, 356-364, 2006.

Kikuchi, T.: VLF phase anomalies associated with substorm, Mem. Nat. Inst. Polar Res. (Special issue), 18, 3-23, 1981.

Kleimenova, N. G., Kozyreva, O. V., Rozhnoi, A. A., and Solovieva, M. S.: Variation in the VLF signal parameters on the AustraliaKamchatka radio path during magnetic storms, Geomagnet. Aeronomy, 44, 3, 354-361, 2004.

Mareev, E. A., Iudin, D. I., and Molchanov, O. A.: Mosaic source of internal gravity waves associated with seismic activity, in: Seismo Electromagnetics: Lithosphere-Atmosphere-Ionosphere Coupling, (edited by: Hayakawa, M. and Molchanov, O.), TERRAPUB, Tokyo, 249-253, 2002.

Molchanov, O. A. and Hayakawa, M.: Subionospheric VLF signal perturbations possibly related to earthquakes, J. Geophys. Res., 103, 17489-17504, 1998.

Molchanov, O. A.: On the origin of low-and middle-latitude ionospheric turbolance, Phys. Chem. Earth., 29, 559-567, 2004.

Molchanov, O., Rozhnoi, A., Solovieva, M., Akentieva, O., Berthelier, J. J., Parrot, M., Lefeuvre, F., Biagi, P. F., Castellana, L., and Hayakawa, M.: Global diagnostics of the ionospheric perturbations related to the seismic activity using the VLF radio signals collected on the DEMETER satellite, Nat. Hazards Earth Syst. Sci., 6, 745-753, doi:10.5194/nhess-6-745-2006, 2006.

Moldovan, A. S., Moldovan, I. A., Placinta, A. O., and Biagi, P. F.: The Romanian VLF/LF monitoring system as a part of the INFREP International Network, Geophys. Res. Abstr., 12, EGU2010-1205-3, 2010.

Rozhnoi, A. A., Solovieva, M. S., Molchanov, O. A., Hayakawa, M., Maekawa, S., and Biagi, P. F.: Anomalies of LF signal during seismic activity in November-December 2004, Nat. Hazards Earth Syst. Sci., 5, 657-660, doi:10.5194/nhess-5-657-2005, 2005.

Rozhnoi, A. A., Solovieva, M. S., Molchanov, O. A., Chebrov, V., Voropaev, V., Hayakawa, M., Maekawa, S., and Biagi, P. F.: Preseismic anomaly of LF signal on the wave path Japan-Kamchatka during November 2004, Phys. Chem. Earth, 31, 422-427, 2006 a.

Rozhnoi, A., Solovieva., M. S., Molchanov, O. A., Hayakawa, M., Maekawa, S. and Biagi, P. F.: Sensitivity of LF signal to global ionosphere and atmosphere perturbations in the network of stations, Phys. Chem. Earth, 31, 409-415, $2006 \mathrm{~b}$.

Rozhnoi, A., Molchanov, O., Solovieva, M., Gladyshev, V., Akentieva, O., Berthelier, J. J., Parrot, M., Lefeuvre, F., Hayakawa, M., Castellana, L., and Biagi, P. F.: Possible seismo-ionosphere perturbations revealed by VLF signals collected on ground and on a satellite, Nat. Hazards Earth Syst. Sci., 7, 617-624, doi:10.5194/nhess-7-617-2007, 2007. 